\title{
DESIGN OF WHEELCHAIR USING FINGER OPERATION WITH IMAGE PROCESSING ALGORITHMS
}

\author{
Chhaya.G.Patil ${ }^{1}$, Sayali.K.Gharge ${ }^{2}$, Sonal.V.Modhave ${ }^{3}$, Y.S.Angal ${ }^{4}$ \\ ${ }^{1,2,3}$ Student, ${ }^{4}$ HOD Department of Electronics \& Telecommunication Engineering, JSPM's BSIOTR(W), Pune, 412207, \\ Maharashtra, India.
}

\begin{abstract}
This paper may be helpful in making the living of handicapped people easy which will be helpful for the handicapped people to drive their wheelchair through figure operation. It is an opportunity to be a handicapped as independent. System comprises of integration of finger operation using digital image processing and embedded technology.According to the World Health Organization (WHO), between the 7 and 10\% of the population worldwide suffer from some physical disability. In Latin-America the physically disabled are estimated in 55 million people, which represent the $9 \%$ of its total population. This census indicates that the most common disability is motor, followed by blindness, deafness, intellectual and language. All alone, the motor-disabled achieve 20 million in Latin-America and there is an anticipated continued growth due to increasing aging, longevity and accident related injuries. Wheelchairs make up a significant portion of the mobility assistive devices in use for those. Since the 1930s, the design concept of a wheelchair has been the same: a main frame, two large rear wheels and two small front wheels called casters. This basic design has been used to develop many of today' wheelchairs by applying slight design modifications to produce lighter, more durable and more comfortable wheelchairs for people who use them on a daily basis. Finally it means that is affordable and beneficial by the people.
\end{abstract}

Keywords: Digital Image Processing, Embedded Technology, Finger operated Wheelchair

\section{INTRODUCTION}

Wheelchair has always helped the disabled in moving from one place to another, but some find it uncomfortable for controlling it. Initially wheelchairs make up a significant portion of the mobility assistive devices in use with the design concept of a wheelchair has been the same: a main frame, two large rear wheels and two small front wheels called casters. [4]This basic design has been used to develop many of today's wheelchairs by applying slight design modifications to produce lighter, more durable and more comfortable wheelchairs for people who use them on a daily basis. The current wheelchairs are mostly button driven, and cannot fully meet the need of the disabled and elder people. Whose autonomies are seriously affected by decline in their motor function and cognitive performance this is the survey done by WHO in 2002. This census indicates that the most common disability is motor, followed by blindness, deafness, intellectual and language. [1]Still various hands-free human machine interface have been developed for the disabled with the operations of head and tongue motion, speech, pushbutton, eye tracking but the proposed paper represent the image algorithm based finger operated wheelchair which is controlled by finger operations.[6]Use of this proposed technology is very much user friendly and requires very less muscle movement form the user.[9]An IR obstacle detection unit can be used which is fixed to the wheelchair to avoid possible collision. In such a way proposed technology shows embedded system and Digital image Processing together work for movement of wheelchair.

\subsection{Problem Statement}

In this era of the technology and healthcare are fast growing, there are still considerable amounts of physically challenged and elderly who find it difficult to move around in their house. But often quadriplegics and tetraplegia people will find it uncomfortable or somewhat difficult to control the wheelchair manually and will go in search of an external aid. Thus there is a need for an improved method of Navigation. [2][8]One such method proposed here is the gesture based navigation in which simple hand gestures forms the input to the system which is processed, recognized and used for navigating the wheelchair. By this method, the user with disability will find it comfortable for indoor navigation and does not need an external aid. 


\section{PROPOSED METHODOLOGY}

\subsection{Block Diagram}

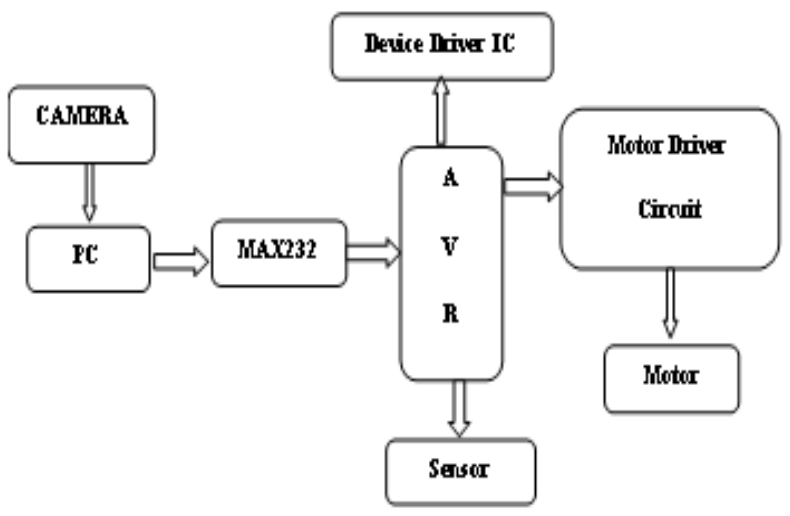

Fig1: Block Diagram of Finger Operated Wheelchair

\subsection{Description}

\subsubsection{Camera}

Camera is used for image link and its permit computer for video conference station .Mostly webcam used for World Wide Web.We can use webcam for capturing image input from finger of user. For this we decide certain circumference of the camera. Webcam consist lens, image sensor, support electronics. The camera is connected to frame of the Wheelchair. The camera has to provide the User monitoring drive of the wheelchair Capabilities According to the JTAG Standard

\subsubsection{PC}

The image of the patient is received by the camera connected to the PC. In PC image is processed and the signal is given to the microcontroller (AVR) and the motor is drive accordingly

\subsubsection{AVR: Advance Virtual Risc}

The AVR is a modified Harvard architecture 8-bit RISC single chip microcontroller. The AVR was one of the first microcontroller families to use on-chip flash memory for program storage, as opposed to onetime programmable ROM, EPROM, or EEPROM used by other microcontrollers at the time.

- Advanced RISC Architecture

- 131 Powerful Instructions - Most

- Single-clock Cycle Execution

- $32 \times 8$ General Purpose Working Registers

- Fully Static Operation

- High Endurance Non-volatile Memory segments

- 32Kbytes of In-System Self-programmable Flash program memory

- 1024Bytes EEPROM
- 2Kbytes Internal SRAM

- In-System Programming by On-chip Boot Program

- True Read-While-Write Operation

- Programming Lock for Software Security

- JTAG (IEEE std. 1149.1 Compliant) Interface

- Boundary-scan

\subsubsection{MAX 232}

It is used to communicate with AVR and PC via serial communication. The $\mu \mathrm{c}$ uses TTL CMOS technology

\subsubsection{Motors}

For Real time wheelchair stepper motors with appropriate gears is used. For small model motors of $12 \mathrm{~V}$ DC motor are used.

Table1: Operation of Motor

\begin{tabular}{|c|c|c|}
\hline OPERATION & A & B \\
\hline STOP & LOW & LOW \\
\hline CLOCKWISE & LOW & HIGH \\
\hline ANTICLOCKWISE & HIGH & LOW \\
\hline STOP & HIGH & LOW \\
\hline
\end{tabular}

\subsubsection{L293D}

The behavior of motor for various inputs:

- 600MA Output Current Capability per Channel

- 1.2A Peak Output Current (Non repetitive) Per Channel

- Enable Facility

- Over temperature Protection

- Logical "0" Input Voltage up to $1.5 \mathrm{v}$ (High Noise Immunity)

\subsubsection{IR SENSOR}

The main Features of Infrared Sensor:

- Infrared Sensor Output: 4 to $20 \mathrm{~mA}$

- Accuracy: $\pm 1 \%$ of reading or $\pm 1^{\circ} \mathrm{C}$ whichever is greater

- Repeatability: $\pm 0.5 \%$ of reading or $\pm 0.5^{\circ} \mathrm{C}$ whichever is greater

- Emissivity: 0.2 to 1.0 via 4 to $20 \mathrm{~mA}$ input

- Response Time t90: $240 \mathrm{~ms}$ (90\% response)

- Spectral Range: 8 to $14 \mu \mathrm{m}$

- Supply Voltage: $24 \mathrm{~V} \mathrm{dc}(28 \mathrm{~V} \mathrm{dc} \max )$

- Min Sensor Voltage: $6 \mathrm{~V}$ dc

- Max Loop impedance $900 \Omega$ (4 to $20 \mathrm{~mA}$ output)

- Input Impedance: $50 \Omega$ Mechanical 
- Construction: Stainless Steel

- Ambient Temperature Range: 0 to $70^{\circ} \mathrm{C}$ (32 to $\left.158^{\circ} \mathrm{F}\right)$

- Relative Humidity: 95\% max non-condensing

\subsubsection{Algorithm for Movement of Wheelchair:-}

1. Start

2. Grab an image

1. Blurring the image

2. Convert RGB to HSV

3. Take HSV Thresholding

4. Finger detection

5. Gesture recognition

6. END

\subsubsection{Flow Chart:}

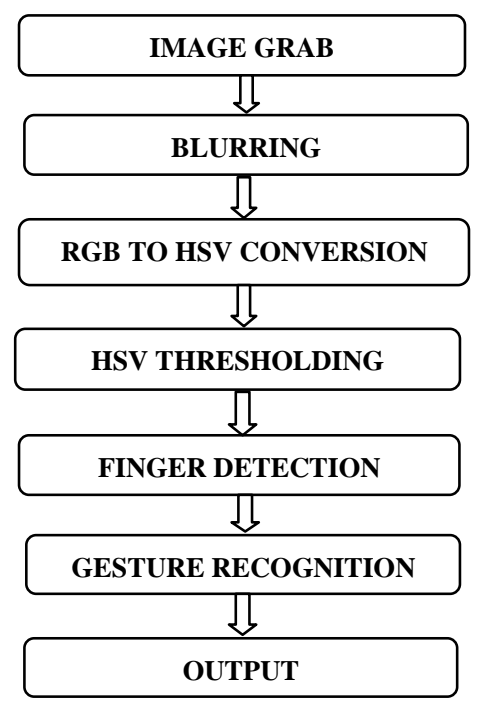

Fig2: Flowchart for Finger Operated Wheelchair

\subsection{Flow Chart Description:}

\subsubsection{Image Grab:}

Select an image for input. An image is capture by camera which is fixed on the frame of Wheelchair. An image is an array, or a matrix, of square pixels arranged in columns and rows. Image can decide by user.

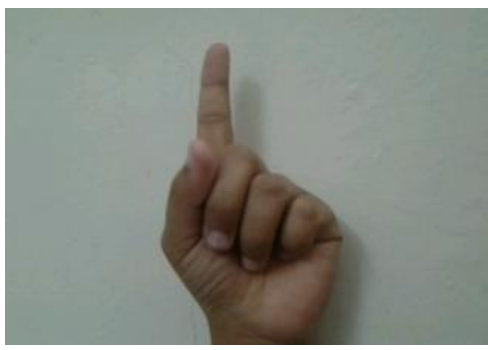

Fig3. An image capture for Forward Operation

\subsubsection{Blurring:}

[7]Blurring means that each pixel in the source image gets spread over and mixed into surrounding pixels. Another way to look at this is that each pixel in the destination image is made up out of a mixture of surrounding pixels from the source image.

\section{Benefits of Blurred Image:}

- Blurring an image reduces the sharpening effect, this makes the detection more accurate.

- It minimizes the Salt \& Pepper noise.

\section{Algorithm for blurring:}

- Traverse through entire input image array.

- Read individual pixel colour value (24-bit).

- Split the colour value into individual R, G and B 8-bit values.

- Calculate the RGB average of surrounding pixels and assign this average value to it.

- Repeat the above step for each pixel.

- Store the new value at same location in output image.

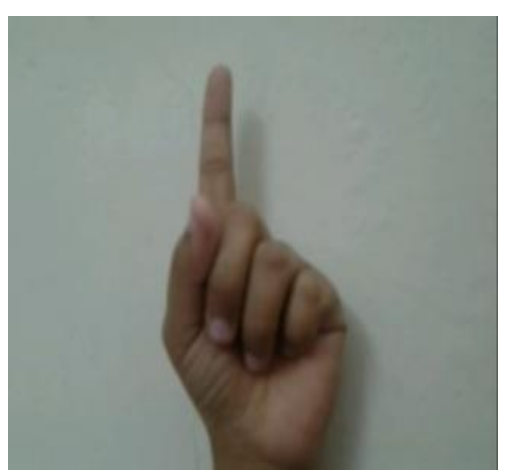

Fig4. Blurred image of Forward Operation

\subsubsection{RGB to HSV:}

There are two main colour spaces are RGB and CMYK. The RGB colour model relates very closely to the way we perceive colour with the $r, g$ and $b$ receptors in our retinas. RGB uses additive colour mixing and is the basic colour model used in television or any other medium that projects colour with light. It is the basic colour model used in computers and for web graphics, but it cannot be used for print production. The secondary colours of RGB - cyan, magenta, and yellow - are formed by mixing two of the primary colours (red, green or blue) and excluding the third colour. Red and green combine to make yellow, green and blue to make cyan, and blue and red form magenta. The combination of red, green, and blue in full intensity makes white. It is basically used for separation of colour from an image. To recognize exact input even it is in colour. 
HSV model give information about:

H- Hue (Dominant colour)

Specify the position of pure colour

S-saturation (Presence of white colour)

Describe the how white the colour is. E.g. pure red is fully saturated; tints of red have saturations less than 1

V-value (Brightness)

Describe intensity of colour can be described as brightness in the colour.

\section{Advantages of HSV Over RGB:-}

- Strong model than RGB because it offers a more intuitive representation of the relationship between colors. .

- HSV select more specific color.

\section{Algorithm for RGB to HSV:-}

- Load image.

- Read each pixel from image

- Separate RGB color for each pixel

- Assign max to value.

- If value equal to zero thenassign hue $=$ saturation $=0$.

- Set pixel in image again.

- If value(Lum) $=0$ then output color is black.

- $\quad$ Find saturation= $255 *($ Max-Min $) /$ value.

- If saturation is zero then assign hue is zero.set pix

- If $\max$ equal to $\mathrm{R}$ then hue $=0+43 *(\mathrm{G}-\mathrm{B}) /(\max -$ $\min )$.

- If $\max$ is equal to $\mathrm{G}$ then hue $=85+43 *(\mathrm{~B}-\mathrm{R}) /(\max -$ $\min )$.

- If $\max$ is equal to $\mathrm{B}$ then hue $=171+43 *(\mathrm{~B}$ $\mathrm{R}) /(\max -\min )$.

- If hue $<0$ then hue $=$ hue +255 .

- $\quad$ Set each pixel again on image.

- HSV Thresholding in done after the conversion.
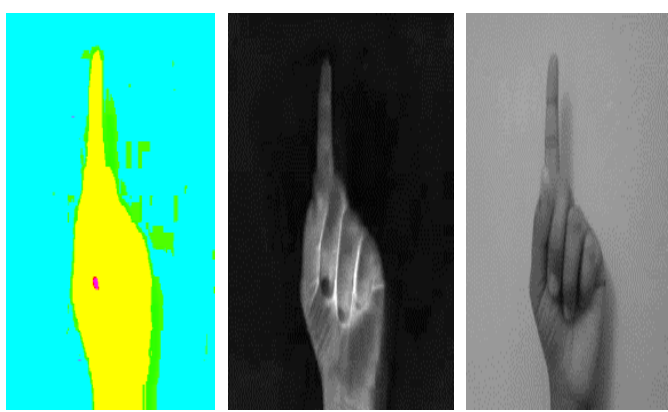

Fig5. HSV images of Forward Operation

\subsubsection{HSV Thresholding:}

Thresholding is the simplest method of image segmentation. From a grayscale image, thresholding can be used to create binary images. During the thresholding process, individual pixels in an image are marked as "object" pixels if their value is greater than some threshold value. This convention is known as threshold above. Variants include threshold below, which is opposite of threshold above.

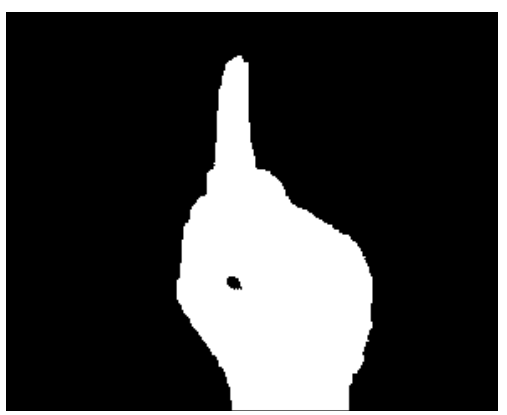

Fig6. Forward Operation image with Thresholding

\subsubsection{Fingure Detection and Recognition:}

After the process of blurring, RGB to HSV conversion followed by thresholding the main and important part of proposed paper is that the finger recognition. The various methods available for this task But we are using computer graphics in which a trigonometric circle is used to recognize the movement of finger.

\section{Algorithm}

- $\quad$ Find width of input

- Recognition of finger

- Find the number of finger which cuts the circumference of circle

- $\quad$ Find out the angle between finger and vertical line

- If Midangle > $135 \&$ midangle < 225 then move Forward

- Midangle $>90 \&$ midangle $<135$ then move left

- Midangle $>225 \&$ midangle $<270$ then move right

- Else stop

- $\quad$ Finger count $==2$ then move reverse. 


\section{RESULTS}

Table 2: Result of wheelchair movement

\begin{tabular}{|c|c|c|c|c|c|}
\hline $\begin{array}{l}\text { Sir } \\
\text { no }\end{array}$ & $\begin{array}{l}\text { Finger } \\
\text { Movement }\end{array}$ & Image & $\begin{array}{l}\text { Motor } \\
\text { Movement }\end{array}$ & $\begin{array}{l}\text { Motor } \\
\text { Movement }\end{array}$ & $\%$ Accuracy \\
\hline & & & Motor A & Motor B & 100 \\
\hline 1 & Forward & & Forward & Forward & 100 \\
\hline 2 & Reverse & & Reverse & Reverse & 100 \\
\hline 3 & Left & & Forward & Reverse & 100 \\
\hline 4 & Right & & Reverse & Forward & 100 \\
\hline 5 & Stop & & $\begin{array}{l}\text { No } \\
\text { Operation }\end{array}$ & $\begin{array}{l}\text { No } \\
\text { Operation }\end{array}$ & 100 \\
\hline
\end{tabular}

\section{CONCLUSIONS}

The main objective of wheelchair to make easy life for handicapped people. It has been observe that the wheelchair essential tool for handicapped people. The wheelchair can be developed according to specific diseases. The AVR microcontroller is used for interfacing to wheelchair. The current driver and motor driver is necessary to set up proper current requirement and driving the motor. Here we take color image of finger recognition input, we can take for gray scale input which gives less accuracy in capturing image.

\section{ACKNOWLEDGEMENTS}

We are thankful to principal of Jspm's (Bhivarabai Sawant Institute of Technology \& Research (W)) Dr.D.M.YADAV for helping us for Image Processing part .We want to extend many thanks to PROF. PRAJAKTA PATIL and workshop session of Bhivarabai Sawant Institute of Technology \& Research.

\section{REFERENCES}

[1]. J.S. Han, Z. Zenn Bien, D.J. Kim, H.E. Lee, and J.S. Kim. Human- machine interface for wheelchair control with EMG and its evaluation. In Proc. of the 25th Annual Int. Conf. of the IEEE Engineering in Medicine \& Biology Society, volume 2, pages 1602-1605. IEEE, 2003.

[2]. P. Jia, H.H. Hu, T. Lu, and K. Yuan. Head gesture recognition for hands-free control of an intelligent wheelchair.
Industrial Robot: An International Journal, 34(1):60-68,2007 [3]. I.Moon, M. Lee, J. Chu, and M. Mun. Wearable EMGbased HCI for electric-powered wheelchair users with motor disabilities. In Proceedings of the 2005 IEEE International Conference on Robotics and Automation, pages 2649-2654. IEEE, 2005.

[4]. R. Cooper, "Wheelchair Selection and Configuration", Demos Medical Publishing, Inc., New York, 1998.

[5]World Health Organization, "The World Health Report 2002: Reducing Risks, Promoting Healthy Life", Geneva, Switzerland, 2002.

[6]. T. Gomi and A. Griffith, "Developing intelligent wheelchairs for the handicapped wheelchair," in Assistive Technology and Artificial Intel- ligence, V. Mittal, H. Yanco, J. Aronis, and R. Simpson, Eds. New York: Springer, 1998, pp. 151-178.

[7]. Gonzalez, Rafael C. \& Woods, Richard E. 2002. Digital Image Processing, second edition Prentice-Hall Pearson

[8]. R. C. Simpson, "Smart wheelchairs: A literature review," J. of Re- habilitation Research \& Development, Vol.42, No.4, pp. 423-436, 2005.

[9]. "An assistive navigation system for wheelchairs based upon mobile robot obstacle avoidance," in Proc. IEEE Int. Conf. Robot. Automation, New York: IEEE Press, 1994, pp. 2018-2022. 


\section{BIOGRAPHIES}

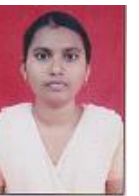

Chhaya Gopal Patil, Department of Electronics \& Telecommunication Engineering, JSPM'S BSIOTR (W), Pune, Maharashtra, India.

Email id-chhayagpatil@gmail.com

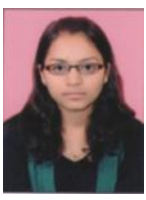

Sayali Kamalakar Gharge, Department of Electronics \& Telecommunication Engineering, JSPM'S BSIOTR(W),Pune, Maharashtra, India. Emailid-gharge.sayali12@gmail.com

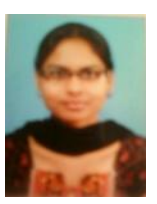

Sonal Vilas Modhave, Department of Electronics \& Telecommunication Engineering, JSPM'S BSIOTR(W), Pune, Maharashtra, India.

Emailid-Smodhave15@gmail.com

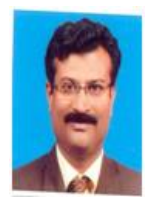

Yogesh S. Angal received the B.E. (Instrumentation) and M.E. (Instrumentation) degrees in 1996 and 2002 respectively from Shri Guru. Gobind Singhji (S.G.G.S) Institute of Engineering and Technology, Vishnupuri, Nanded, Maharashtra State. He has done his research in Speech Signal Processing area. His areas of interest include Digital Signal Processing, Speech Processing, Mechatronics and Control Systems. He is presently Working as HOD of ENTC Department in JSPM'S BSIOTR (W), Pune, India. Emailid-yogeshangal@yahoo.co.in 